Copyright (C 2014 IEEE. Personal use of this material is permitted. Permission from IEEE must be obtained for all other uses, in any current or future media, including reprinting/republishing this material for advertising or promotional purposes, creating new collective works, for resale or redistribution to servers or lists, or reuse of any copyrighted component of this work in other works. 


\title{
Comparative Numerical Studies of Electromechanical Finite Element Vibration Power Harvester Approaches of a Piezoelectric Unimorph
}

\author{
Eziwarman, Mikail F. Lumentut, Member, IEEE, and Ian M. Howard
}

\begin{abstract}
Emerging micro-power harvester research using smart material components shows viable self-powered devices capable of capturing mechanical motion and converting it into useful electrical energy that can be further used to supply electrical voltage into rechargeable power storage via a power management electronic circuit. The micro-power harvesters using piezoelectric materials cover a wide range of applications for powering thin film battery technology and wireless sensor systems that can be used to monitor the health condition of machines and infrastructure and biomedical implant devices. This research focuses on the development of a novel numerical direct method technique with non-orthonormality based on the electromechanical vector transformation for modelling the selfpowered cantilevered piezoelectric unimorph beam under input base excitation. The proposed finite element piezoelectric unimorph beam equations were formulated using Hamiltonian's principle for formulating the global matrices of electromechanical dynamic equations based on the electromechanical vector transformation that can be further employed to derive the electromechanical frequency response functions. This numerical technique was modelled using electromechanical discretisation consisting of mechanical and electrical discretised elements due to the electrode layers covering the surfaces of the piezoelectric structure, giving the single voltage output. The reduced equations are based on the Euler-Bernoulli beam assumption for designing the typical power harvesting device. The proposed finite element models were also compared with orthonormalised electromechanical finite element response techniques, giving accurate results in the frequency domains.
\end{abstract}

\section{INTRODUCTION}

The investigation of piezoelectric power harvesters using mathematical studies plays the important role to model and predict the electrical power output based on the frequency responses and transient time domain behaviour of the structure. Most vibration environments from the machines and infrastructure, including biomechanical human motion give relatively lower amplitude and frequency ranges that can be used for matching the system response from the piezoelectric structure in order to maximise the power output. For this reason, development of analytical studies of the piezoelectric power harvesters has received much attention from researchers. The piezoelectric power harvesters using

Eziwarman, Mikail Lumentut and Ian Howard are with the Laboratory for Dynamic Systems of Smart Structure and Vibration, Department of Mechanical Engineering, Curtin University, Australia

(e-mail: m.lumentut@exchange.curtin.edu.au, eziwarman@yahoo.com, and i.howard@curtin.edu.au). unimorph and bimorph beams have been investigated using novel analytical studies with different parametric case studies [1]-[5]. However, there are minor concerns in developing the numerical studies of the electromechanical power harvesting devices. The development of finite element analysis for modelling piezoelectric active control systems has made major contributions during the last two decades and these can be used to give fundamental concepts and cross-reference numerical studies to the current energy harvesting research studies. The finite element active control analysis using the laminated smart structure system such as coupled piezoelectric and substructure was formulated using the variational principle [6], [7]. More details of the active control system using various numerical methods can be found in the published literature reviews [8]. In the power harvesting research area, novel technical concepts of the finite element methods have shown alternative solution techniques for investigating the self-powered MEMS devices using complex geometry of the laminated piezoelectric beams with electrode layers combined with the external circuit components. Only a few of the finite element selfpowered harvester research studies have been investigated by researchers during the last six years [9]-[11].

Although many researchers have investigated various numerical techniques for developing the governing electromechanical finite element dynamic equations including other numerical solutions, this paper presents novel numerical direct method techniques of the electromechanical finite element analysis for investigating power harvesting frequency response. Here, the research focus is to present derivations of the finite element equations with nonorthonormality based on the electromechanical vector transformation in order to develop the electromechanical frequency response functions (FRFs) for the voltage, current and power harvesting. The proposed studies are compared with the series form of the multimode FRFs derived from the orthonormalised global scalar forms of the electromechanical finite element equations. Finally, the numerical case studies using the numerical convergence studies and frequency responses based on the resistive shunt circuit are presented and discussed, where the results obtained from the two numerical solution techniques show good agreement.

\section{Finite Element MODELING OF PIEZOELECTRIC ENERGY HARVESTER}

In this section, the piezoelectric unimorph beam under base excitation and variable load resistance as shown in Fig. 1 is 
utilised for modelling the electromechanical finite element vibration energy harvester.

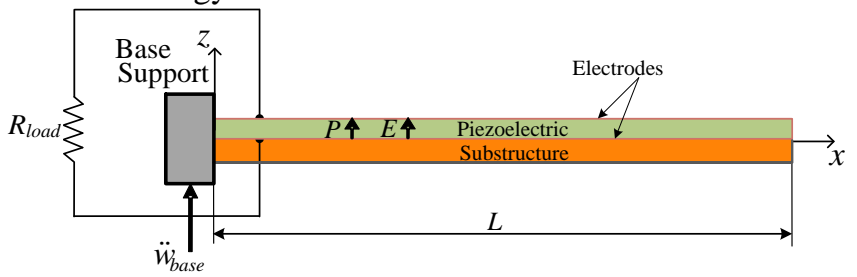

Fig. 1. Piezoelectric unimorph beam connected with load resistance under base motion

\section{A. Electromechanical coupled finite element formulation}

The linear constitutive equations of the Lead Zirconate Titanate (PZT) beam can be formulated with 3-1 mode of piezoelectric constant operation and 3-3 effect of piezoelectric permittivity as,

$$
\begin{gathered}
\boldsymbol{T}_{1}^{(2)}=\bar{c}_{11}^{(2)} \boldsymbol{S}_{1}^{(2)}-e_{31} \boldsymbol{E}_{3}, \\
\boldsymbol{D}_{3}=e_{31} \boldsymbol{S}_{1}^{(2)}+\varepsilon_{33}^{S} \boldsymbol{E}_{3} .
\end{gathered}
$$

The linear-elastic constitutive relation for the substructure can also be formulated as,

$$
\boldsymbol{T}_{1}^{(1)}=\bar{c}_{11}^{(1)} \boldsymbol{S}_{1}^{(1)},
$$

where the parameters $\boldsymbol{T}, \boldsymbol{S}, \boldsymbol{E}$ and $\boldsymbol{D}$ represent stress, strain, electric field, and electric displacement, respectively. Moreover, coefficients $c, e$, and $\varepsilon$ indicate elastic constant, piezoelectric coefficient, and permittivity at constant strain, respectively. The superscript 1 and 2 represent substructure and piezoelectric layers, respectively.

The first-order Hermite interpolation function of the unimorph beam can be formulated to give,

$$
\boldsymbol{w}(x, t)=\boldsymbol{\Phi}^{e}(x) \boldsymbol{u}^{e}(t),
$$

where parameters $\boldsymbol{\Phi}$ and $\boldsymbol{u}$ indicate the elemental displacement vector and shape function for each node. The strain-displacement relationship in terms of the vector displacement for the unimorph beam can be expressed as,

$$
\boldsymbol{S}(x, t)=-z \Psi^{e}(x) \boldsymbol{u}^{e}(t),
$$

where $\Psi$ is the second order differential shape function for the strain displacement and $\boldsymbol{u}$ is the vector mechanical displacement.

The discretised electric field $\boldsymbol{E}$ induced by the strain field creates the electrical potential $\phi$ over the piezoelectric element and is assumed to be linear along the thickness of the element. The electric field component as a function of the electrical potential with negative gradient operator can be represented as,

$$
\boldsymbol{E}_{3}=-\nabla \boldsymbol{\phi}^{e}(z, t)=-\boldsymbol{\Omega}^{e}(z) \boldsymbol{\nu}^{(e)}(t) .
$$

The stress field in the partial differential shape function can be expressed by substituting Eq. (4) and Eq. (5) into Eq. (1a) and Eq. (2) to give,

$$
\begin{gathered}
\boldsymbol{T}_{1}^{(2)}=-z \overline{\boldsymbol{c}}_{11}^{(2)} \boldsymbol{\Psi}^{e}(x) \boldsymbol{u}^{e}(t)+e_{31} \boldsymbol{\Omega}(z) \boldsymbol{v}^{e}(t), \\
\boldsymbol{T}_{1}^{(1)}=-z \overline{\boldsymbol{c}}_{11}^{(1)} \boldsymbol{\Psi}^{\boldsymbol{e}}(x) \boldsymbol{u}^{\boldsymbol{e}}(t) .
\end{gathered}
$$

The direct mode for electric displacement vector of the piezoelectric component can be formulated by substituting Eqs. (4)-(5) into Eq. (1b) and this can be expressed as,

$$
\boldsymbol{D}_{3}=z e_{31} \Psi^{e}(x) \boldsymbol{u}^{e}(t)-\varepsilon_{33}^{S} \Omega^{e}(z) \boldsymbol{v}^{e}(t) .
$$

\section{B. Extended Hamiltonian principles}

Electromechanical piezoelectric unimorph power harvesting response using finite element modelling can be formulated using the Hamiltonian principle to give,

$$
\int_{t_{1}}^{t_{2}}[\delta(K E-P E+W E)+\delta W F] \mathrm{d} t=0 .
$$

The total kinetic and potential energy terms can be defined as,

$$
\begin{gathered}
K E=\int_{V_{1}} \frac{1}{2} \rho^{(1)} \dot{\boldsymbol{w}}^{T} \dot{\boldsymbol{w}} \mathrm{d} V^{(1)}+\int_{V_{2}} \frac{1}{2} \rho^{(2)} \dot{\boldsymbol{w}}^{T} \dot{\boldsymbol{w}} \mathrm{d} V^{(2)}, \\
P E=\int_{V_{1}} \frac{1}{2} \boldsymbol{S}_{1}^{(1)^{T}} \boldsymbol{T}_{1}^{(1)} \mathrm{d} V^{(1)}+\int_{V_{2}} \frac{1}{2} \boldsymbol{S}_{1}^{(2)^{T}} \boldsymbol{T}_{1}^{(2)} \mathrm{d} V^{(2)} . \\
W E=\int_{V_{2}}^{1} \frac{1}{2} \boldsymbol{E}_{3}^{T} \boldsymbol{D}_{3} d V^{(2)} .
\end{gathered}
$$

The non-conservative work for the system due to input base excitation and electrical charge output can be written as,

$$
\delta W F=\boldsymbol{F} \delta \boldsymbol{w}+\boldsymbol{q} \delta \boldsymbol{v}
$$

Parameters $\rho$ and $V$ indicate mass density and volume, respectively. Superscript $T$ denotes the transpose matrix and over-dot represents the differentiation with respect to time.

The Hamiltonian's principle for energy harvesting can then be written as,

$$
\left[\begin{array}{l}
\int_{V_{1}} \rho^{(1)} \delta \dot{\boldsymbol{w}}^{T} \dot{\boldsymbol{w}} \mathrm{d} V^{(1)}+\int_{V^{(2)}} \rho^{(2)} \delta \dot{\boldsymbol{w}}^{T} \dot{\boldsymbol{w}} \mathrm{d} V^{(2)} \\
-\int_{V^{(1)}} \delta \boldsymbol{S}_{1}^{(1)^{T}} \boldsymbol{c}_{11}^{(1)} \boldsymbol{S}_{1}^{(1)} \mathrm{d} V^{(1)}-\int_{V^{(2)}} \delta \boldsymbol{S}_{1}^{(2)^{T}} \boldsymbol{c}_{11}^{(2)} \boldsymbol{S}_{1}^{(2)} \mathrm{d} V^{(2)} \\
+\int_{V^{(2)}} \delta \boldsymbol{S}_{1}^{(2)^{T}} e_{31} \boldsymbol{E} \mathrm{d} V^{(2)}+\int_{V^{(2)}} \delta \boldsymbol{E}^{T} e_{31} \boldsymbol{S}_{1}^{(2)} \mathrm{d} V^{(2)} \\
+\int_{V^{(2)}} \delta \boldsymbol{E}^{T} \varepsilon_{33}^{S} \boldsymbol{S}_{1}^{(2)} \mathrm{d} V_{2}+\boldsymbol{F} \delta \boldsymbol{w}+\boldsymbol{q} \delta \boldsymbol{v}
\end{array}\right] \mathrm{d} t=0
$$

The expression given from Eq. (13) can be further solved by substituting Eqs. (3)-(7) to give the local element matrices of electromechanical dynamic equations by including Rayleigh damping as,

$$
\left[\begin{array}{cc}
\boldsymbol{M}^{e} & 0 \\
0 & 0
\end{array}\right]\left\{\begin{array}{l}
\ddot{\boldsymbol{u}}^{e} \\
\ddot{\boldsymbol{v}}^{e}
\end{array}\right\}+\left[\begin{array}{cc}
\boldsymbol{C}^{e} & 0 \\
\boldsymbol{P}_{\theta}^{e^{T}} & \boldsymbol{P}_{\mathbf{D}}
\end{array}\right]\left\{\begin{array}{c}
\dot{\boldsymbol{u}}^{e} \\
\boldsymbol{v}^{e}
\end{array}\right\}+\left[\begin{array}{cc}
\boldsymbol{K}^{e} & \boldsymbol{P}_{\theta}^{e} \\
0 & 0
\end{array}\right]\left\{\begin{array}{l}
\boldsymbol{u}^{e} \\
\boldsymbol{v}^{e}
\end{array}\right\}=\left\{\begin{array}{l}
\boldsymbol{F}^{e} \\
\boldsymbol{i}^{e}
\end{array}\right\}
$$

where:

$$
\begin{aligned}
& \boldsymbol{M}^{e}=\sum_{n l=1} \int_{V^{(n l)}} \rho^{(n l)} \boldsymbol{\Phi}^{e}(x)^{T} \boldsymbol{\Phi}^{e}(x) \mathrm{d} V^{(n l)}, \\
& \boldsymbol{K}^{e}=\sum_{n l=1} \int_{V^{(n l)}} z^{2} \overline{\boldsymbol{c}}_{11}^{(n l)} \boldsymbol{\Psi}^{e}(x)^{T} \boldsymbol{\Psi}^{e}(x) \mathrm{d} V^{(n l)}, \\
& \boldsymbol{P}_{\theta}^{\mathrm{e}}=-\sum_{n p=1_{V}} \int_{V^{(n p)}} z e_{31}^{(n p)} \boldsymbol{\Psi}^{e}(x)^{T} \boldsymbol{\Omega}^{e}(z) \mathrm{d} V^{(n p)}, \\
& \boldsymbol{P}_{\theta}^{e^{T}}=-\sum_{n p=1} \int_{V^{(n p)}} z e_{31}^{(n p)} \boldsymbol{\Omega}^{e}(z)^{T} \boldsymbol{\Psi}^{e}(x) \mathrm{d} V^{(n p)},
\end{aligned}
$$




$$
\begin{gathered}
\boldsymbol{P}_{D}^{e}=-\sum_{n p=1} \int_{V_{n p}} \varepsilon_{33}^{(n p)} \boldsymbol{\Omega}^{\mathrm{e}}(\mathrm{z})^{T} \boldsymbol{\Omega}^{e}(z) \mathrm{d} V_{n p}, \\
\boldsymbol{Q}^{e}=\sum_{n l=1} \int_{A^{(n l)}} \rho^{(n l)} \Phi^{e}(x)^{T} \mathrm{~d} A_{n l}, \boldsymbol{F}^{\mathbf{e}}=-\boldsymbol{Q}^{\mathrm{e}} \ddot{w}_{b}(t) .
\end{gathered}
$$

\section{Global matrices of electromechanical dynamic equations based on the electromechanical vector transformation}

The generalised dynamic equations derived from Eq. (14) can be formulated into the global matrix forms to give,

$$
\begin{aligned}
& \underset{\left(n_{m} \times n_{m}\right)}{\boldsymbol{M}} \underset{\left(n_{m} \times 1\right)}{\ddot{\boldsymbol{u}}(t)}+\underset{\left(n_{m} \times n_{m}\right)}{\boldsymbol{C}} \underset{\left(n_{m} \times 1\right)}{\dot{\boldsymbol{u}}(t)}+\underset{\left(n_{m} \times n_{m}\right)}{\boldsymbol{K}} \underset{\left(n_{m} \times 1\right)}{\boldsymbol{u}(t)}+\underset{\left(n_{m} \times n_{e}\right)}{\boldsymbol{P}_{\theta}} \underset{\left(n_{e} \times 1\right)}{\boldsymbol{v}(t)}=\underset{\left(n_{m} \times 1\right)}{\boldsymbol{F}}
\end{aligned}
$$

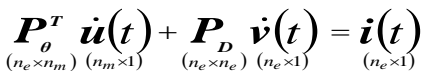

where $\boldsymbol{M}$ is global mass matrix, $\boldsymbol{K}$ is global stiffness matrix and $\boldsymbol{C}$ is Rayleigh damping. Other parameters $\boldsymbol{P}_{\theta}, \boldsymbol{P}_{D}$, and $\boldsymbol{F}$ indicate global electromechanical coupling matrices, global capacitance matrices, global mechanical forces, respectively. Moreover, variable $\boldsymbol{i}$ is global current output, $\boldsymbol{u}$ is global mechanical coordinate, and $v$ is global voltage output.

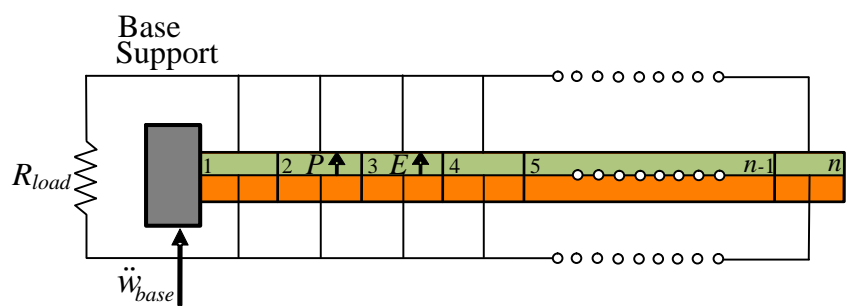

Fig. 2. Electromechanical discretisation of piezoelectric unimorph element

In practical standpoint, the electrode layers cover the top and bottom of the piezoelectric material where the physical system only has two wires connected to the two electrode layers resulting in one single voltage output as shown in Fig. 1. However, for analysing global finite element equations as shown in Fig. 2, the modelling shows different scenario since the whole structure of the piezoelectric beam consists of the mechanical discretised element and the electrical discretised element (electromechanical discretisation). For this case, the generalised multi-output electrical voltage from each element can be formulated in terms of Kirchhoff's voltage law (KVL) as,

$$
\begin{gathered}
v_{1}=v_{2}=\ldots v_{m}=v_{p} \text { or } \\
\boldsymbol{v}=\left\{\begin{array}{lllll}
1 & 1 & \ldots & 1
\end{array}\right\}^{T} v_{p}=\boldsymbol{\vartheta}^{T} v_{p},
\end{gathered}
$$

and electrical current using Kirchhoff's current law (KCL) related to global current matrix $\boldsymbol{i}$ can also be formulated as,

$$
i_{p_{1}}+i_{p_{2}}+\ldots+i_{p s}=i_{p}=\boldsymbol{\vartheta} \boldsymbol{i} .
$$

Given the characteristic discretised system, equation (14) needs to be modified since the generalised voltage outputs in the global coordinate still show distinct values. To alleviate this issue, the electromechanical transformation $\vartheta$ from Eq. (16) can be used to multiply with the electromechanical coupling matrices taken from the fourth term of the first expression in Eq. (15). The operational form can be formulated as,

$$
\underset{\left(n_{m} \times n_{\mathrm{e}}\right)}{\boldsymbol{P}_{\boldsymbol{\theta}}} \underset{\left.n_{e} \times 1\right)}{\boldsymbol{\vartheta}^{T}} v_{p}=\underset{\left(n_{m} \times 1\right)}{\boldsymbol{\Theta}} v_{p} .
$$

In addition, the second expression of the global matrices from Eq. (15b) must also be modified by multiplying the electromechanical vector transformation $\vartheta$ at both sides of Eq. (15b) and introducing the electrical vector transformation at the second term of Eq. (15b), the results of which can be formulated as,

$$
\boldsymbol{\vartheta} \boldsymbol{P}_{\boldsymbol{\theta}}^{T} \dot{\boldsymbol{u}}+\boldsymbol{9} \boldsymbol{P}_{D} \boldsymbol{\vartheta}^{T} \dot{v}_{p}=\boldsymbol{\vartheta} \boldsymbol{i}
$$

Reformulating Eq. (15a) using Eq. (18) and altering Eq. (19) using Eq. (17) and the current expression $i_{p}=v_{p} / R_{\text {load }}$, the new formation of the electromechanical finite element equation can be written as,

$$
\begin{gathered}
\boldsymbol{M} \ddot{\boldsymbol{u}}+\boldsymbol{C} \dot{\boldsymbol{u}}+\boldsymbol{K u}+\boldsymbol{\Theta} v_{p}=-\boldsymbol{Q} \ddot{w}_{b} \\
\boldsymbol{\Theta}^{T} \dot{\boldsymbol{u}}+\frac{v_{p}}{R_{\text {load }}}+C_{p} \dot{v}_{p}=0
\end{gathered} .
$$

Note that the modified electromechanical coupling $\boldsymbol{\vartheta} \boldsymbol{P}_{\boldsymbol{\theta}}{ }^{T}$ is equivalent to the transformed electromechanical coupling $\boldsymbol{\Theta}^{T}$ and the capacitance matrix $\boldsymbol{\vartheta} \boldsymbol{P}_{D} \boldsymbol{\vartheta}^{T}=C_{p}$ is equivalent to the trace (tr) of the global capacitance matrix $\boldsymbol{P}_{D}$.

\section{SOlution of Finite ElEMENT EQUATIONS}

\section{A. Current model of electromechanical frequency response equations}

The piezoelectric response under base excitation can be assumed to be harmonic. Therefore, solutions of the mechanical displacement and voltage responses can be formulated as $\boldsymbol{u}=\boldsymbol{a} e^{j \omega t}$ and $v_{p}=b e^{j \omega t}$. Here, variable $\boldsymbol{a}$ is the amplitude of the base translation and $b$ is the amplitude of the harmonic voltage and $\omega$ is the driving frequency. The assumed solutions can be substituted into Eq. (20) to formulate the electromechanical FRFs.

The multi-mode voltage FRFs can be formulated after simplifying the derivations to give,

$$
\begin{aligned}
\frac{v_{p}}{-\omega^{2} w_{b} e^{j \omega t}} & =\left[\begin{array}{l}
j \underset{(1 \times 1)}{j \omega} \underset{(1 \times 1)}{C_{p}-\frac{1}{R_{\text {load }}}-j \omega \underset{\left(1 \times n_{m}\right)}{\boldsymbol{\Theta}}} \\
\times\left[\begin{array}{c}
\underset{\left(n_{m} \times n_{m}\right)}{\boldsymbol{K}}-\underset{\left(n_{m} \times n_{m}\right)}{\omega^{2} \boldsymbol{M}}+\underset{\left(n_{m} \times n_{m}\right)}{j \omega \boldsymbol{C}}
\end{array}\right]^{-1} \underset{\left(n_{m} \times 1\right)}{\boldsymbol{\Theta}}
\end{array}\right]^{-1} . \\
& \times j \omega \underset{\left(1 \times n_{m}\right)}{\boldsymbol{\Theta}^{\mathrm{T}}}\left[\underset{\left(n_{m} \times n_{m}\right)}{\boldsymbol{K}}-\underset{\left(n_{m} \times n_{m}\right)}{\omega^{2} \boldsymbol{M}}+\underset{\left(n_{m} \times n_{m}\right)}{j \boldsymbol{C}}\right]^{-1} \underset{\left(n_{m} \times 1\right)}{\boldsymbol{Q}}
\end{aligned}
$$

The multi-mode FRFs of the electric current output related to the input base transverse excitation can also be formulated by dividing the voltage response with the load resistance to yield, 


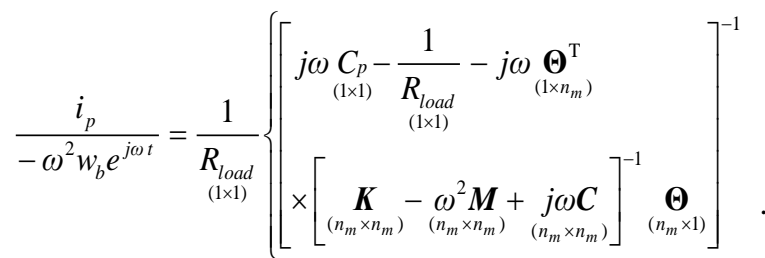

$$
\begin{aligned}
& \left.\times j \omega \underset{\left(1 \times n_{m}\right)}{\boldsymbol{\Theta}^{\mathrm{T}}}\left[\underset{\left(n_{m} \times n_{m}\right)}{\boldsymbol{K}}-\underset{\left(n_{m} \times n_{m}\right)}{\omega^{2} \boldsymbol{M}}+\underset{\left(n_{m} \times n_{m}\right)}{j \omega \boldsymbol{C}}\right]^{-1} \underset{\left(n_{m} \times 1\right)}{\boldsymbol{Q}}\right\}
\end{aligned}
$$

The multi-mode power output FRFs can also be formulated as,

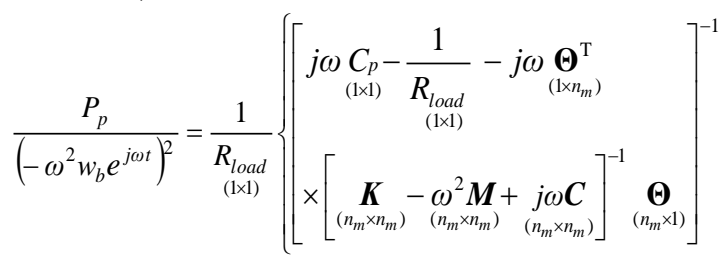

$$
\begin{aligned}
& \left.\times j \omega \underset{\left(1 \times n_{m}\right)}{\boldsymbol{\Theta}^{\mathrm{T}}}\left[\underset{\left(n_{m} \times n_{m}\right)}{\boldsymbol{K}}-\underset{\left(n_{m} \times n_{m}\right)}{\omega^{2} \boldsymbol{M}}+\underset{\left(n_{m} \times n_{m}\right)}{j \omega \boldsymbol{C}}\right]^{-1} \underset{\left(n_{m} \times 1\right)}{\boldsymbol{Q}}\right\}^{2}
\end{aligned}
$$

B. Orthonormalised electromechanical finite element modelling

Other new techniques for solution of electromechanical finite element vibration equations as shown in section 2 can be developed using the orthonormalised scalar forms of electromechanical dynamic equations. These techniques can be useful in making comparisons with the proposed current model. The solution form of Eq. (15) can be stated using time-dependent displacement in terms of the normalised modal vector and time-dependent displacement generalised coordinate as,

$$
\boldsymbol{u}(t)=\boldsymbol{\varphi}_{1} \boldsymbol{a}_{1}(t)+\boldsymbol{\varphi}_{2} \boldsymbol{a}_{2}(t)+\ldots+\boldsymbol{\varphi}_{m-1} \boldsymbol{a}_{m-1}(t)+\boldsymbol{\varphi}_{m} \boldsymbol{a}_{m}(t)=\varphi \boldsymbol{a}(t)
$$

where the normalised modal matrix can be formulated as,

$$
\boldsymbol{\varphi}=\boldsymbol{U}\left(\boldsymbol{U}^{\boldsymbol{T}} \boldsymbol{M U}\right)^{-\frac{1}{2}}
$$

Parameters $\boldsymbol{\varphi}$ and $\boldsymbol{U}$ represent a set of the normalised modal matrix and generalised eigenvector, respectively. The eigenvector reduced from the undamped mechanical dynamic equation, is a mechanical shape of natural mode that can be used to formulate the orthogonality property. The undamped mechanical dynamic equation depends on the mechanical properties from the stiffness and mass matrices.

Substituting Eq. (24) into Eq. (15) and premultiplying the result by $\varphi^{T}$ in order to diagonalise the mass, stiffness and damping matrices and simplifying the numerical technique, provides the formulations,

$$
\begin{gathered}
\boldsymbol{\varphi}^{T} \boldsymbol{M} \dot{\boldsymbol{a}}(t)+\boldsymbol{\varphi}^{T} \boldsymbol{C} \boldsymbol{\varphi} \dot{a}(t)+\boldsymbol{\varphi}^{T} \boldsymbol{K} \boldsymbol{\varphi} \boldsymbol{a}(t)+\boldsymbol{\varphi}^{T} \boldsymbol{P}_{\boldsymbol{\theta}} \boldsymbol{v}(t)=\boldsymbol{\varphi}^{T} \boldsymbol{F} \\
\boldsymbol{P}_{\theta}^{T} \dot{\boldsymbol{a}}(t)+\boldsymbol{P}_{\boldsymbol{D}} \dot{\boldsymbol{v}}(t)=\boldsymbol{i}(t)
\end{gathered}
$$

or simplifying Eq. (26) becomes,

$$
\begin{gathered}
\ddot{\boldsymbol{a}}(t)+2 \zeta \omega \dot{\boldsymbol{a}}(t)+\omega^{2} \boldsymbol{a}(t)+\hat{\boldsymbol{P}}_{\boldsymbol{\theta}} \boldsymbol{v}(t)=-\hat{\boldsymbol{Q}}_{\ddot{\boldsymbol{w}}_{\text {base }}}(t), \\
\hat{\boldsymbol{P}}_{\theta}^{T} \dot{\boldsymbol{a}}(t)+\boldsymbol{P}_{D} \dot{\boldsymbol{v}}(t)=\boldsymbol{i}(t) .
\end{gathered}
$$

Orthonormalised parameters from Eq. (27) can be stated as,

$$
\begin{gathered}
\varphi^{T} M \varphi=I, \quad \varphi^{T} K \varphi=\omega^{2}, \\
\varphi^{T} C \varphi=\alpha\left(\varphi^{T} M \varphi\right)+\beta\left(\varphi^{T} K \varphi\right)=\alpha I+\beta \omega^{2}=2 \zeta \omega, \\
\hat{P}_{\theta}=\varphi^{T} P_{\theta}, \quad \hat{P}_{\theta}^{T}=P_{\theta}^{T} \boldsymbol{\varphi}, \quad \hat{Q}=\varphi^{T} Q .
\end{gathered}
$$

It should be noted that equations (28a) and (28b) represent the orthonormality property of mechanical dynamic equations, the results of which indicate diagonal matrices.

Equation (27) can be further simplified into global scalar form of the electromechanical dynamic equations in order to formulate the series form of the multimode frequency response function. In this case, the first form of the discretised electromechanical piezoelectric dynamic equation can be formulated for the multi degree of freedom (multimode) system $r=1,2,3, \ldots ., N D O F$ in terms of the number of normalised piezoelectric elements $s=1,2,3, \ldots ., N E L P$ as,

$$
\begin{gathered}
\ddot{a}_{r}(t)+2 \zeta_{r} \omega_{r} \dot{a}_{r}(t)+\omega_{r}{ }^{2} a_{r}(t)+\sum_{s=1}^{N E L P} \hat{P}_{r s} v_{s}(t)=-\hat{Q}_{r} \ddot{w}_{\text {base }}(t), \\
r=1,2, \ldots, N D O F .
\end{gathered}
$$

The second form of the discretised electromechanical piezoelectric dynamic equation can be formulated as,

$$
\begin{aligned}
\sum_{r=1}^{N D O F} \hat{P}_{s r} \dot{a}_{r}(t)+P_{D s} \dot{v}_{s}(t) & =i_{p s}(t), \\
s & =1,2, \ldots, N E L P .
\end{aligned}
$$

As stated previously, the piezoelectric surface is covered evenly by the electrode layers. Therefore, electromechanical equations should be based on the electromechanical discretisation as shown in Fig. 1b. Since the proposed equations are formulated in the global scalar form, here the internal parallel connection in terms of the electrical discretised elements using the Kirchhoff's voltage law (KVL) and Kirchhoff's current law (KCL) must be formulated in the scalar form as,

$$
\begin{gathered}
v_{1}(t)=v_{2}(t)=\ldots=v_{s}(t)=v(t), \\
i_{p 1}(t)+i_{p 2}(t)+\ldots .+i_{p s}(t)=i_{p}(t) .
\end{gathered}
$$

Voltage output related to the external load resistance can be formulated as,

$$
v(t)=i_{p}(t) R_{\text {load }}
$$

To formulate multimode frequency response functions of the distributed piezoelectric unimorph, the first step is to modify the first term of Eq. (30) algebraically corresponding with the number of the normalised piezoelectric elements. Second, employing Eqs. (31) and (32) into the equations obtained from the first step. Third, applying Eq. (31) into Eq. (29). Fourth, the results obtained from the second and third steps can be algebraically solved using Laplace transforms giving the superposition matrix form. The first superposition 
form of the voltage multimode FRFs series can be formulated after simplifying as,

$$
\frac{v(j \omega)}{-\omega^{2} w_{b} e^{j \omega t}}=\frac{\sum_{r=1}^{N D O F} \frac{\hat{Q}_{r} j \omega \sum_{s=1}^{N E L P} \hat{P}_{s r}}{\omega_{r}{ }^{2}-\omega^{2}+j 2 \zeta_{r} \omega_{r} \omega}}{\sum_{s=1}^{N E L P} j \omega P_{D s}-\frac{1}{R_{\text {load }}}-\sum_{r=1}^{N D O F} \frac{j \omega \sum_{s=1}^{N E L P} \hat{P}_{s r} \sum_{s=1}^{N E L P} \hat{P}_{r s}}{\omega_{r}{ }^{2}-\omega^{2}+j 2 \zeta_{r} \omega_{r} \omega}} \cdot
$$

The series form of the multimode FRF for the electric current output related to the input base transverse acceleration can be stated as,

$$
\frac{i_{p}(j \omega)}{-\omega^{2} w_{b} e^{j \omega t}}=\frac{\frac{1}{R_{\text {load }}} \sum_{r=1}^{N D O F} \frac{\hat{Q}_{r} j \omega \sum_{s=1}^{N E L P} \hat{P}_{s r}}{\omega_{r}{ }^{2}-\omega^{2}+j 2 \zeta_{r} \omega_{r} \omega}}{\sum_{s=1}^{N E L P} j \omega P_{D s}-\frac{1}{R_{\text {load }}}-\sum_{r=1}^{N D O F} \frac{j \omega \sum_{s=1}^{N E L P} \hat{P}_{s r} \sum_{s=1}^{N E L P} \hat{P}_{r s}}{\omega_{r}{ }^{2}-\omega^{2}+j 2 \zeta_{r} \omega_{r} \omega}} .
$$

The power harvesting multimode FRF related to the input transverse acceleration can be formulated as,

$$
\frac{P_{p}(j \omega)}{\left(-\omega^{2} w_{b} e^{j \omega t}\right)^{2}}=\left[\frac{\frac{1}{\sqrt{R_{\text {load }}}} \sum_{r=1}^{N D O F} \frac{\hat{Q}_{r} j \omega \sum_{s=1}^{N E L P} \hat{P}_{s r}}{\omega_{r}{ }^{2}-\omega^{2}+j 2 \zeta_{r} \omega_{r} \omega}}{\sum_{s=1}^{N E L P} j \omega P_{D s}-\frac{1}{R_{\text {load }}}-\sum_{r=1}^{N D O F} \frac{j \omega \sum_{s=1}^{N E L P} \hat{P}_{s r} \sum_{s=1}^{N E L P} \hat{P}_{r s}}{\omega_{r}{ }^{2}-\omega^{2}+j 2 \zeta_{r} \omega_{r} \omega}}\right]^{2} .
$$

\section{RESUlT AND DisCUSSION}

In this section, the convergence study and the first two modes of voltage, current and power FRFs are discussed using the proposed numerical methods. Here, the geometry and material properties of the piezoelectric unimorph beam are given in table 1 . Note that the input base excitation onto the structure was set to be $1 \mathrm{~m} / \mathrm{s}^{2}$.

TABle I. MATERIAL PROPERTIES OF UNIMORPH POWER HARVESTER

\begin{tabular}{ll}
\hline Dimensional & Parameter \\
\hline Length of the beam, $L(\mathrm{~mm})$ & 60 \\
Width of the beam, $b(\mathrm{~mm})$ & 6 \\
$\begin{array}{l}\text { Thickness of the substructure, } h_{s}(\mathrm{~mm}) \\
\text { Thickness of the PZT, } h_{p}(\mathrm{~mm})\end{array}$ & 0.5 \\
Young's Modulus of the substructure, $\bar{c}_{11}^{(1)}(\mathrm{GPa})$ & 105 \\
Young's Modulus of the PZT, $\bar{c}_{11}^{(2)}(\mathrm{GPa})$ & 66 \\
Mass Density of the substructure layer, $\rho^{(1)}\left(\mathrm{kg} / \mathrm{m}^{3}\right)$ & 9000 \\
Mass Density of the PZT layer, $\rho^{(2)}\left(\mathrm{kg} / \mathrm{m}^{3}\right)$ & 7800 \\
Piezoelectric constant, $d_{31}(\mathrm{pm} / \mathrm{V})$ & -190 \\
Permittivity, $\varepsilon_{33}^{T}(\mathrm{~F} / \mathrm{m})$ & 15.93 \\
\hline
\end{tabular}

The convergence study shows the very important aspect that can be used to achieve better accuracy of the numerical results. Here, the purpose of presenting the convergence study as shown in Fig. 3 is to show the trend of the frequency responses for each mode based on the increasing number of elements. The results shows that increasing the number of elements changes the resonance frequency value for each mode. After 10 or 15 elements only, very minor frequency changes were seen for the first three modes. Therefore, the unimorph with 50 discretised elements should be sufficient for modelling and investigating the FRFs of the first two modes as shown in the next stage.

As previously mentioned, the proposed numerical direct method techniques with non-orthonormality were compared with the orthonormalised electromechanical finite element response techniques. The results show that electromechanical frequency responses of the system have similarities between two different methods. Starting with the electrical voltage FRF as shown in Fig. 4, the trends of the two numerical methods for the electrical voltage amplitudes under variable load resistance show very good agreement and the amplitudes increase from short to open circuit load resistances, followed by a frequency shift. This occurs especially at the first mode where the second mode of frequency response seems to stay at a constant resonance frequency with increasing amplitudes. By inspecting the two enlarged views from Fig. 4, the first mode of the electrical voltage FRF with short circuit resonance frequency shows $89.38 \mathrm{~Hz}$ and the frequency shifts to $90.94 \mathrm{~Hz}$ for the open circuit resonance frequency.

Moreover, the current FRF under variable load resistance as shown in Fig. 5 appears to have the opposite trend to that of the voltage FRFs where the current magnitudes decrease monotonically with increasing load resistance values. Again, the two different numerical methods consistently show good agreement. It is important to note here that the actual short circuit load resistance approaches to zero, that, however, cannot be used for making the comparison between theoretical and experimental studies since the voltage FRF will be zero and the displacement response cannot be identified [2]. From that reason, the short circuit load resistance can be achieved at the certain lowest values such as $100 \Omega$, as presented in this paper.

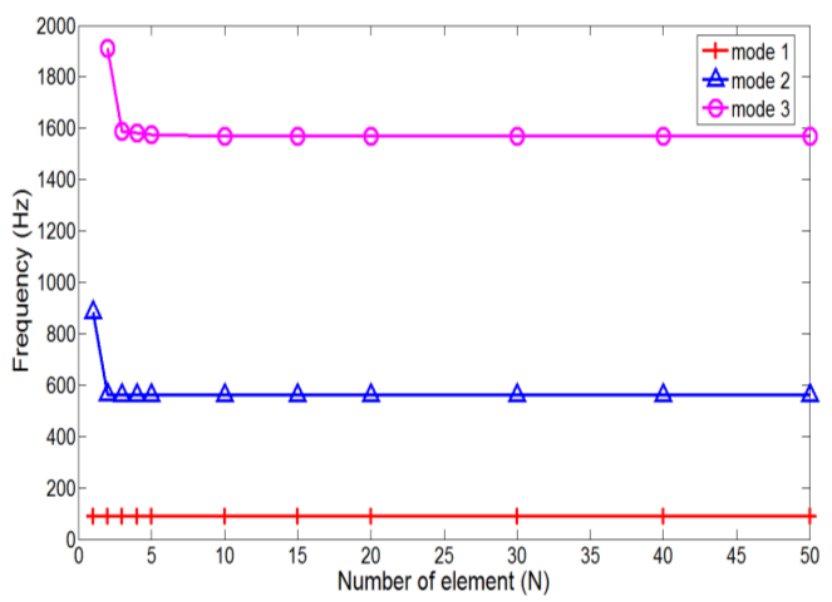

Fig. 3. Convergence studies of the resonance frequency based on the number of element 


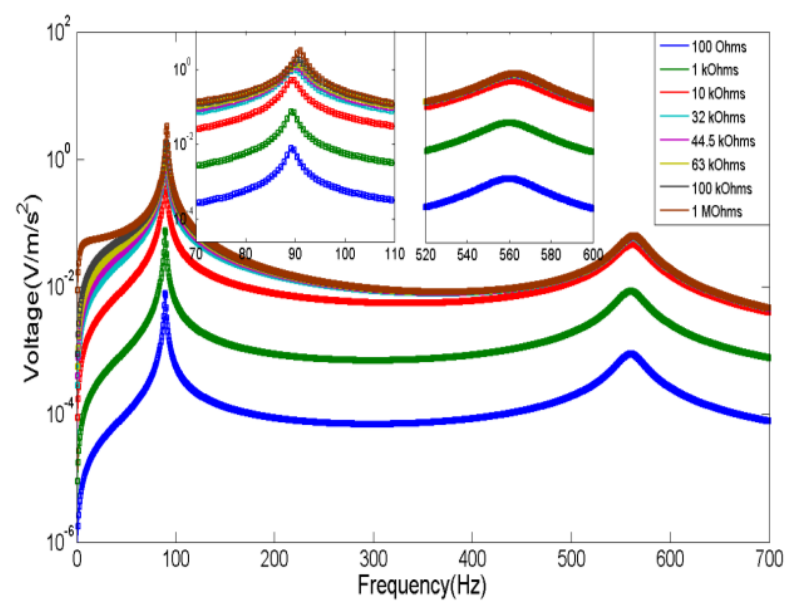

Fig. 4. Electrical voltage FRFs with 50 discretised elements: solid line (non-orthornormalised) and round dot (orthonormalised method)

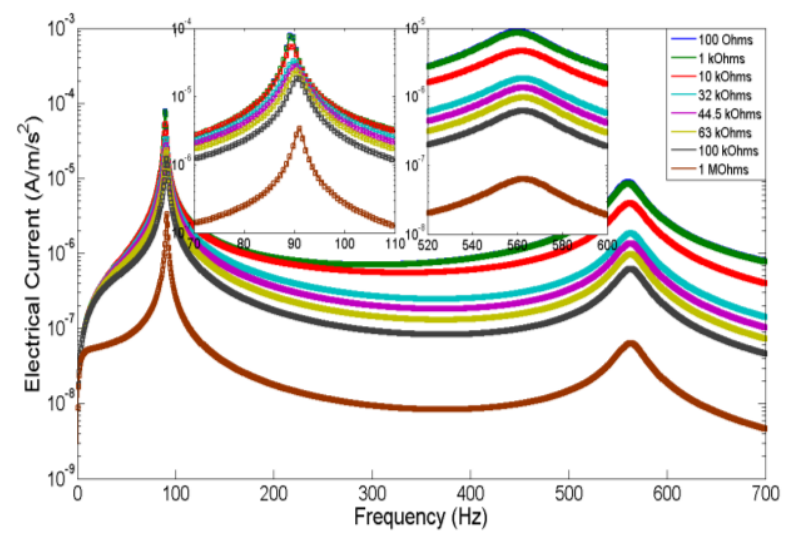

Fig. 5. Electrical current FRFs with 50 discretised elements: solid line (non-orthornormalised) and round dot (orthonormalised method)

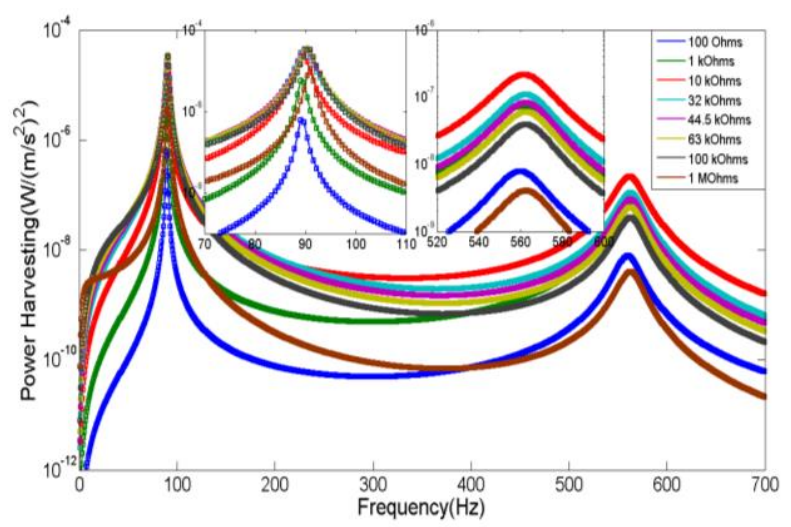

Fig. 6. Electrical power FRFs with 50 discretised elements: solid line (non-orthornormalised) and round dot (orthonormalised method)

In aforementioned statement, the electromechanical frequency responses of the voltage and current have the opposite behaviour when load resistances change where the maximum voltage amplitude can be seen in the highest load resistance whereas the maximum current amplitude can be achieved at the lowest load resistance (short circuit). However, such situation clearly does not appear in the power output FRFs as shown in Fig. 6 where the maximum power output at the first mode does not necessarily depend on the short or open circuit load resistance. For this case, the maximum power output response appeared when the resistance value coincides with load resistance of $32 \mathrm{k} \Omega$ at the frequency of $89.92 \mathrm{~Hz}$. Meanwhile, the maximum power output at the second mode appears at the resonance of 561.5 $\mathrm{Hz}$ when the load resistance approaches to $10 \mathrm{k} \Omega$.

\section{CONCLUSION}

This paper discussed novel numerical techniques for modeling a piezoelectric unimorph power harvester derived by using the matrix electromechanical dynamic equations based on the electromechanical vector transformation. The proposed model was also compared using the orthonormalised global scalar forms of electromechanical dynamic equations. The result shows that the multimode frequency responses of the voltage, current and power under variable load resistance using the two numerical methods show good agreement. The benefit of using the numerical techniques is to analyse the multi-level piezoelectric power harvesters with complex geometry and different scalability of MEMS structure where such techniques can alleviate the tedious solutions required by the analytical techniques.

\section{REFERENCES}

[1] M.F. Lumentut and I.M. Howard, "Analytical modeling of selfpowered electromechanical piezoelectric bimorph beams with multidirectional excitation," Int. J. Smart Nano Mater., vol. 2, pp. 134175, 2011.

[2] M.F. Lumentut and I.M. Howard, "Electromechanical piezoelectric Power Harvester Frequency Response Modelling Using Closed-Form Boundary Value Methods," IEEE/ASME Trans. on Mech., vol. 19, no 1, pp. 32 - 44, 2014.

[3] M.F. Lumentut and I.M. Howard, "Analytical and experimental comparisons of electromechanical vibration response of a piezoelectric bimorph beam for power harvesting," Mech. Syst. Signal Process, vol. 36, no. 1, pp.66-86, 2013.

[4] A.M. Wickenheiser and E. Garcia, " Broadband vibration-based energy harvesting improvement through frequency up-conversion by magnetic excitation," Smart Mater. Struct. vol. 19, 2010, art. no. 065020.

[5] F. Goldschmidtboeing and P.Woias, "Characterization of different beam shapes for piezoelectric energy harvesting," J. Micromech. Microeng., vol. 18, 2008, art. no. 104013.

[6] J.M. Moita, I.F.P. Correia and C.M.M. Soares, "Active control of adaptive laminated structures with bounded piezoelectric sensors and actuators," Comp. Struct. vol. 82 1349-58, 2008.

[7] O. Thomas, J.-F Deü and J. Ducarne , "Vibrations of an elastic structure with shunted piezoelectric patches: efficient finite element formulation and electromechanical coupling coefficients," Int. J. Numer. Methods Engng. vol. 8 235-268, 2009.

[8] A. Benjeddou, "Advances in piezoelectric finite element modeling of adaptive structural elements: a survey', Comp.Struct., 2000, 76, pp. 347-363.

[9] M.F. Lumentut, K.K. Teh and I.M. Howard, "Computional FEA model of a coupled piezoelectric sensor and plate structure for energy harvesting', Aust. J. Mech. Engng, 5, pp. 199-208, 2008.

[10] C. Jr. DeMarqui, A. Erturk and D.J. Inman, "An electromechanical Finite Element Model for piezoelectric Energy Harvester Plates," J. Sound. Vib., vol. 327, pp. 29-25, 2009.

[11] N.G. Elvin and A.A. Elvin, "A coupled finite element-circuit simulation model for analyzing piezoelectric energy generators," J. Intel. Mater. Syst. Struct., vol. 20, pp. 587-595, 2009. 